\title{
Breakdown of the continuum limit approximation to the discrete scattering events and its influence on the electric field autocorrelation functions of transmitted light
}

\author{
Miloš Šormaz, ${ }^{1, *}$ Mathias Reufer, ${ }^{2}$ Andreas C. Völker, ${ }^{2}$ and Klaus Simon ${ }^{1}$ \\ ${ }^{1}$ Laboratory for Media Technology, Swiss Federal Laboratories for Materials Science and Technology (EMPA), \\ Überlandstrasse 129, 8600 Dübendorf, Switzerland \\ ${ }^{2}$ LS Instruments AG, Passage du Cardinal 1, 1700 Fribourg, Switzerland
}

(Received 19 August 2014; published 3 November 2014)

\begin{abstract}
The complexity of modeling light propagation in turbid media can be significantly simplified if one assumes it to be diffusive. This is, however, only valid after the light has traveled a sufficient distance so that the diffusion equation can be employed. So far, there has been no reliable way to determine this distance, despite the fact that the assumption is often applied in optics. The discrete nature of scattering events plays an important role in modeling propagation of weakly scattered light, so a continuum equation such as the diffusion equation cannot be used to describe this process. Electric field autocorrelation functions $g_{1}(\tau)$ of light transmitted through turbid colloidal samples are measured using diffusing wave spectroscopy and compared to Monte Carlo simulations in order to obtain a better estimation of the continuum limit. The two methods to calculate $g_{1}(\tau)$ from the simulated photon trajectories are compared; the first assumes the continuum limit by using the path-length distributions of photon trajectories, while the second considers the square momentum transfers and therefore accurately calculates $g_{1}(\tau)$ even if the detected signal is composed of weakly scattered light. The results of the two methods are used to determine the lengths of the shortest diffuse photon trajectories; they grow with the sample thickness and scattering anisotropy.
\end{abstract}

DOI: 10.1103/PhysRevA.90.053802

PACS number(s): 42.25.Dd, 87.10.Rt, 87.64.-t, 42.25.Ja

\section{INTRODUCTION}

The diffusion equation is often used to model radiative transfer in turbid media [1-3], such as the propagation of light in a colloidal system. It assumes a continuum limit and therefore cannot describe discrete scattering events on length scales comparable to or shorter than the transport mean free path $l^{*}$ [4]. Typically, a ratio of $L / l^{*}>10$ is suggested to ensure validity of the continuum limit approximation, where $L$ is the sample thickness. This assumption is, however, rather arbitrary and, in many cases, it is not clear if the continuum approximation can be applied and how large the error might be if it is not fully valid. Specifically, in backscattering, it is difficult to determine the validity of the continuum limit. Therefore, it is important to understand the conditions under which the diffusion approximation can be applied and how its limits are affected by sample properties and experimental setup.

Diffusing wave spectroscopy (DWS) [5-8] is a popular method that relies on the validity of the continuum limit. The intensity correlation function of photons that are scattered by particles in a homogenous medium allows calculating the mean-square displacement of the particles. It was successfully applied in fundamental research to study colloidal dynamics, nondiffusive Brownian motion [9], samples with correlation between the scattering particles [10], and to study the structure and dynamics of three-dimensional foams [11]. Furthermore, it was used to study the dynamics in the human cortex [12], diagnostic microflow [13], the viscoelasticity of complex fluids [14-16], and the effects of blood flow and extravascular tissue shearing [17].

\footnotetext{
*Corresponding author: milos.sormaz@gmail.com
}

In order to expand the application range of DWS beyond the continuum limit, several extensions were proposed $[18,19]$. In [18], light propagation was modeled with the telegrapher equation to account for ballistic transport between successive scattering events. Furthermore, the probability densities of photon path lengths (determined by solving the timedependent radiative transfer equation) and scattering events along a given path were introduced in [19] to account for the contribution of very long paths with few scattering events that are not captured by the standard diffusion approximation. Geometrical requirements for DWS experiments were presented in [20]. The discrete nature of scattering particles was taken into account in [21] by abandoning the continuum limit approximation and instead considering the shape of the photon trajectories. This has allowed accurate calculations of the autocorrelation functions of a backscattered light.

In this paper, the electric field autocorrelation functions $g_{1}(\tau)$ of light transmitted through cuvettes containing samples of colloidal particles dispersed in water are determined experimentally with DWS and compared to simulations obtained with SCATTER3D [22,23]. The software package SCATTER3D allows modeling the experimental conditions in great detail. It uses Monte Carlo simulation to describe the transport of light through a medium where individual scattering events are calculated using Lorenz-Mie theory. These simulations allow the investigation of the continuum limit approximation when different ratios of $L / l^{*}$, media with different scattering properties, and different polarization detection schemes are considered. We vary $L / l^{*}$ in order to study the transition from diffuse (large $L / l^{*}$ ) to nondiffuse (small $L / l^{*}$ ) transport of light for which the continuum limit is not a valid approximation. This is achieved by using cuvettes of different interior thicknesses $(L=1,2,5,10 \mathrm{~mm})$ with a constant value of $l^{*}$ throughout the study. 


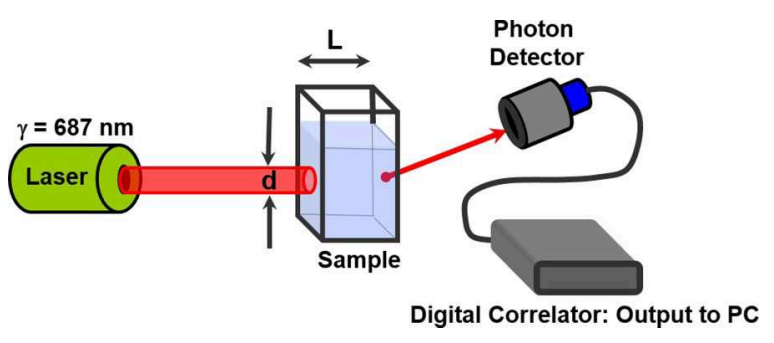

FIG. 1. (Color online) Setup of DWS experiment.

This work reveals an estimate of the minimal photon path length for which the continuum limit is valid. Moreover, the dependence of these paths on the experimental setup and sample under study is revealed.

\section{THEORETICAL BACKGROUND}

The diffusion equation is a continuum limit of a randomwalk description of light transport [24] that allows straightforward computation of the path-length distribution $P(s)$ for some experimental setups. The photon path of length $s$ can be described as a random walk with step size $l^{*}$. The transport mean free path $l^{*}$ is a measure of the typical distance that light needs to travel before it becomes diffuse. It is connected to the scattering mean free path $l$ via $l / l^{*}=1-\langle\cos (\theta)\rangle$, where $\theta$ is the scattering angle. If for the considered experimental conditions (in particular, the chosen $L / l^{*}$ ) the detected light is diffuse (the continuum limit can be assumed), then the electric field autocorrelation function $g_{1}(\tau)$ in a DWS experiment, shown in Fig. 1, can be calculated as

$$
g_{1}(\tau)=\int_{0}^{\infty} P_{F}(s) e^{-\left(2 \tau / \tau_{0}\right) s / l^{*}} d s,
$$

where $P_{F}(s)$ is the angular average histogram of the pathlength distribution. This corresponds to a hemispherical detector which collects all the light emerging from a detection spot, regardless of its propagation direction. Here, $\tau$ and $\tau_{0}=$ $1 /\left(D k^{2}\right)$ are the lag time and the characteristic diffusion time (i.e., the time it takes a particle to move a distance of the order of a wavelength $\lambda$ ), respectively. In the case of particles with radius $r$ in a Newtonian solvent with viscosity $\eta$, the diffusion coefficient $D$ is given by the Stokes-Einstein equation, $D=$ $k_{B} T /(6 \pi \eta r)$. Here, $k_{B}$ is the Boltzmann constant and $T$ is the temperature. For samples with polydisperse particles, one has to replace $\tau_{0}$ and $l^{*}$ in Eq. (1) by $\tau_{\mathrm{eff}}$ and $l_{\mathrm{eff}}^{*}$,

$$
\begin{aligned}
\tau_{\text {eff }} & =\frac{1}{D_{\text {eff }} k^{2}}, \\
1 / l_{\text {eff }}^{*} & =\sum_{i} \frac{1}{l_{i}^{*}}, \\
D_{\text {eff }} & =l_{\text {eff }}^{*} \sum_{i} \frac{D_{i}}{l_{i}^{*}},
\end{aligned}
$$

where $i$ is the index of every species (particles of different size) in the sample.

However, experimental constraints often require low ratios $L / l^{*}$ (e.g., $\left.<10\right)$ and, as a result, a large portion of detected light is not diffuse. For weakly scattered light, one needs a more precise description and it seems straightforward to take into account the shape of the photon paths. This approach was followed by Middleton et al. [21] who calculated $g_{1}(\tau)$ based on the distribution of the sums of square momentum transfers $P_{F}(y)$, where $y=\sum_{j=1}^{n} q_{j}^{2}$. This quantity is calculated from the momentum transfers $q_{\mathrm{j}}=2 k \sin \left(\theta_{\mathrm{j}} / 2\right)$ collected along the path of length $s$. Here, $\theta_{\mathrm{j}}$ are the corresponding scattering angles and $k=2 \pi / \lambda$ is the wave number. For polydisperse samples, this approach can be extended to

$$
\Gamma=\sum_{\mathrm{j}=1}^{n} q_{\mathrm{j}}^{2} D_{\mathrm{j}},
$$

where $D_{\mathrm{j}}$ is the diffusion coefficient of the $j$ th scattering particle. Equation (3) allows the calculation of $g_{1}(\tau)$ as

$$
g_{1}(\tau)=\int_{0}^{\infty} P_{F}(\Gamma) e^{-\Gamma \tau} d \Gamma .
$$

In contrast to $s, \Gamma$ considers the shape of a photon trajectory since it depends on the scattering angles $\theta_{\mathrm{j}}$. This is particularly important if the detected signal is composed of weakly scattered light whose trajectories of the same length $s$ can have significantly different $\Gamma$. The continuum limit is thus only valid if photon trajectories are long enough to contain a large number of scattering events such that their particular shape is no longer important. In the continuum limit approximation $(n=s / l)$ and assuming monodisperse particles, Eq. (3) simplifies to $\Gamma_{\text {th }}=\left\langle q^{2}\right\rangle D n=2 k^{2} D s / l^{*}$, where we used the relation $2\left\langle\sin ^{2}(\theta / 2)\right\rangle=[1-\langle\cos (\theta)\rangle]=$ $l / l^{*}$. This result demonstrates that for diffuse light, the distributions $P_{F}(s)$ and $P_{F}(\Gamma)$ are the same, except for a scaling factor of $2 k^{2} D / l^{*}$.

\section{EXPERIMENTAL SETUP AND MATERIALS}

The measurements were done with a DWS RheoLab (LS Instruments AG, Fribourg, Switzerland). Cuvettes made of optical glass (refractive index 1.5) with interior width of $10 \mathrm{~mm}$ and different thicknesses $L=1,2,5,10 \mathrm{~mm}$ were used. The cuvette containing the sample under study is illuminated by the uniform collimated laser beam with diameter of $d=8 \mathrm{~mm}$ and wavelength $\gamma=687 \mathrm{~nm}$, as depicted in Fig. 1. The incident light is vertically linearly polarized with the Stokes vector $\mathbf{I}=(I, Q, U, V)=(1,-1,0,0)$. Both copolarized and cross-polarized transmitted light, whose detection schemes are denoted throughout the paper as VV and VH, respectively, are detected from a spot on the cuvette wall of $0.9 \mathrm{~mm}$ diameter. Note that for $L=5$ and $10 \mathrm{~mm}$ cuvettes, the $L / d$ ratio is smaller than suggested in [20] $(d>5 L)$; i.e., the illumination spot is not a good enough approximation of an infinitely wide beam for the two thickest cuvettes considered here. This causes deviations between the measured $g_{1}(\tau)$ and those calculated using Eq. (A1), which is derived assuming laterally infinite beam and cuvette.

The studied samples are made of polystyrene spheres dispersed in water with radius probability density function $\operatorname{PDF}(r)$ and mean value $\bar{r}$ obtained by static light scattering. The scattering intensity of a strongly diluted sample was measured in an angular range of $20^{\circ}$ to $150^{\circ}$ relative to toluene using a goniometer system from LS Instruments AG equipped 
with a HeNe laser with $633 \mathrm{~nm}$ wavelength. The resulting form factors were fitted with a Gaussian distributed $\operatorname{PDF}(r)$ using Lorenz-Mie theory. Best fits were found using a mean radius $\bar{r}=227 \mathrm{~nm}$ with standard deviation $\sigma=12 \mathrm{~nm}$ (PS454), $\bar{r}=100 \mathrm{~nm}$ and $\sigma=4 \mathrm{~nm}(\mathrm{PS} 200)$, and $\bar{r}=480 \mathrm{~nm}$ and $\sigma=10 \mathrm{~nm}$ (PS960). The considered range of particle sizes $[\bar{r}-6 \sigma, \bar{r}+6 \sigma]$ in all simulations is divided into 100 bins. For the simulations, we used a refractive index of 1.587 for the polystyrene particles and 1.332 for the solvent (water). The particle concentration was adjusted such that all samples have the same value of $l^{*}=300 \mu \mathrm{m}$. All experiments were performed at the temperature $T=25^{\circ} \mathrm{C}$ at which water has the viscosity $\eta=0.89 \mathrm{mPa}$.

\section{SIMULATIONS}

The simulation setup in SCATTER3D matches the experimental conditions used by the DWS Rheolab and consists of a virtual cuvette, modeled as a nonabsorbing medium, surrounded by air. The polarization gating implementation in SCATTER3D is explained in [22]. The Stokes vectors of all detected photons are first expressed in the laboratory coordinate system and then multiplied by the Mueller matrices of a linear polarizer with vertical transmission (copolarized light is detected), or horizontal transmission (cross-polarized light is detected). After the multiplications, the copolarized and cross-polarized light are given as $I-Q$ and $I+Q$, respectively. The orientation of the axes that define the laboratory coordinate system is the same as of those used to define the incident Stokes vector.

Effective variables in Eq. (2) are calculated following an iterative procedure; i.e., for a given $1 / \mu_{\mathrm{eff}}^{*}=l_{\mathrm{eff}}^{*}=l^{*}$, the transport coefficient $\mu_{i}^{*}=N^{i} \sigma_{s}^{i}\left(1-g^{i}\right)$ of every species (particles of different $r$ ) is calculated until $\epsilon=\left(\mu_{\mathrm{eff}}^{*}-\right.$ $\left.\sum_{i} \mu_{i}^{*}\right) / \mu_{\mathrm{eff}}^{*}<1 \%$. Here, $i=[1,100]$ is, as defined in Eq. (2), the bin index in $\operatorname{PDF}(r)$, while $g^{i}$ and $\sigma_{s}^{i}$ are the anisotropy factor of the scattering phase function and the scattering cross section of particles with radius $r$, respectively. At every iteration, the number density of each species, denoted as $N^{i}$, is changed according to $\operatorname{PDF}(r)$ until $\epsilon<1 \%$. The transport mean-free-path lengths $l_{i}^{*}=1 / \mu_{i}^{*}$ of all species, which are used in Eq. (2), were calculated in this way. The scattering phase functions of all samples are calculated using LorenzMie theory implemented in SCATTER3D. The found values are $D_{\text {eff }} \approx 1.1 \times 10^{-6} \mathrm{~mm}^{2} / \mathrm{s}$ and $\tau_{\text {eff }} \approx 6.2 \mathrm{~ms}$ (PS454), $D_{\text {eff }} \approx 2.4 \times 10^{-6} \mathrm{~mm}^{2} / \mathrm{s}$ and $\tau_{\text {eff }} \approx 2.8 \mathrm{~ms}$ (PS200), and $D_{\text {eff }} \approx 5.1 \times 10^{-7} \mathrm{~mm}^{2} / \mathrm{s}$ and $\tau_{\text {eff }} \approx 13 \mathrm{~ms}$ (PS960). The effective anisotropy factors of the scattering phase functions calculated as

$$
g_{\text {eff }}=\frac{\int g^{i} \operatorname{PDF}(r) \sigma_{s}^{i} d r}{\int \operatorname{PDF}(r) \sigma_{s}^{i} d r}
$$

amount to $g_{\text {eff }} \approx 0.76$ (PS454), $g_{\text {eff }} \approx 0.27$ (PS200), and $g_{\text {eff }} \approx 0.91$ (PS960).

The polydispersity of the scattering particles is implemented in SCATTER3D as it was done in [25]; photons traveling through a sample experience scattering events on particles with size set by the $\operatorname{PDF}(r)$. The scattering mean free path $l=l^{*}\left(1-g_{\text {eff }}\right)$ does not change during the simulation. As a consequence, the difference compared to the light transport in monodisperse solution is only in the fact that the particle size is different for each scattering event. To reduce the calculation time, the scattering amplitude functions $S_{1}$ and $S_{2}$ calculated with Lorenz-Mie theory [23] were precomputed for each particle size.

In order to find the boundary between diffuse and nondiffuse light and how it is influenced by different scattering particles, a minimum length of diffuse light trajectories was found from the relations between $\Gamma$ and $s$ obtained with the Monte Carlo simulations without modeling cuvette. The average value of $\Gamma$ is calculated for each $P_{F}(s)$ bin as $\langle\Gamma\rangle_{s}=\sum_{p=1}^{m} w_{p} \Gamma_{p} / \sum_{p=1}^{m} w_{p}$. Here, $p$ and $m$ are the index of an individual photon and the number of photons inside the $P_{F}(s)$ bin, respectively. The photon with index $p$, after the polarization gating was applied (i.e., after its Stokes vector in the laboratory coordinate system was multiplied by the Mueller matrix of the corresponding polarizer), has weight $w_{p}=I_{p}-Q_{p}$ (copolarized light detected) or $w_{p}=I_{p}+Q_{p}$ (cross-polarized light detected), and $\Gamma_{p}$. The first two elements of the Stokes vector of a photon are denoted as $I_{p}$ and $Q_{p}$. For diffuse light trajectories, $\Gamma_{\text {th }}=D_{\text {eff }} y_{\text {th }}=D_{\text {eff }} 2 k^{2} s / l^{*}$, while the nonlinear relation between $\Gamma$ and $s$ is expected for nondiffuse light. The amount of nondiffuse light in a detected signal, which depends on the sample thickness and scattering anisotropy, can be determined from the $P_{F}(s)$ distributions for each sample once the length of the shortest diffuse light trajectories is found. The inertia effects of water and scattering particles, not captured by the Monte Carlo simulations, have the strongest influence on the measured $g_{1}(\tau)$ when PS960 is considered. The effect is more pronounced for larger scattering particles and larger $L / l^{*}$. Therefore, the experimental data for PS960 was corrected for inertia effects, as explained in the Appendix.

\section{RESULTS}

The experimental setup of DWS RheoLab is simulated by SCATTER3D to allow direct comparisons between simulated and measured $g_{1}(\tau)$. Figures 2-4 compare measured and simulated $g_{1}(\tau)$ of PS454 in cuvettes with interior thicknesses $L=1,2,5,10 \mathrm{~mm}$. For $L=5$ and $10 \mathrm{~mm}$, only the results for cross-polarized light are shown because they overlap with the results obtained with the copolarized detection channel. This is in agreement with the distributions for $P_{F}(s)$ and $P_{F}(\Gamma)$ for $L=1$ and $L=2 \mathrm{~mm}$ in Fig. 5. Here, in the case $L=2 \mathrm{~mm}$, the distributions for the two polarization states are overlapping, whereas for $L=1$, the peak for the VV geometry is shifted towards smaller $s$ values compared to the $\mathrm{VH}$ geometry. Moreover, the peak position in VV geometry is very close to the value of the cuvette thickness (i.e., $1 \mathrm{~mm}$ ) that leads to an important contribution of photon trajectories with very few scattering events, which conserves the initial polarization state. In contrast, the $\mathrm{VH}$ geometry considers only photons that have changed the polarization state and thus require a minimal number of scattering events that leads to a peak shifted to larger $s$ values. The distributions of the two polarization states of the other sample thicknesses [ $2 \mathrm{~mm}$ shown in Fig. 5(b); 5 and $10 \mathrm{~mm}$ not shown] are overlapping and, as a result, the obtained $g_{1}(\tau)$ are insensitive to the polarization detection scheme. This 


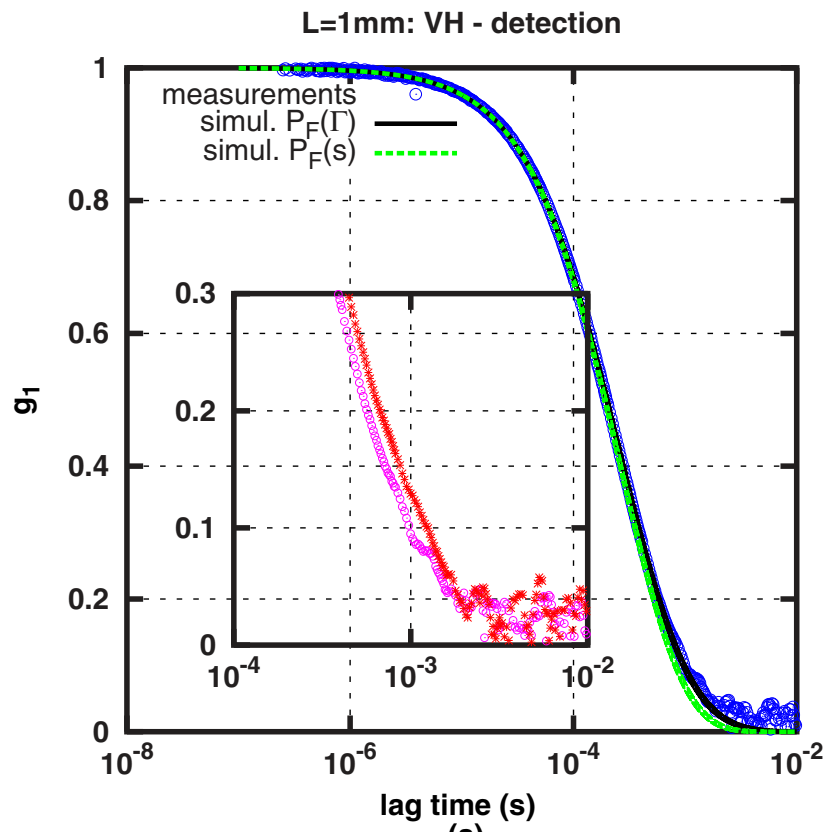

(a)

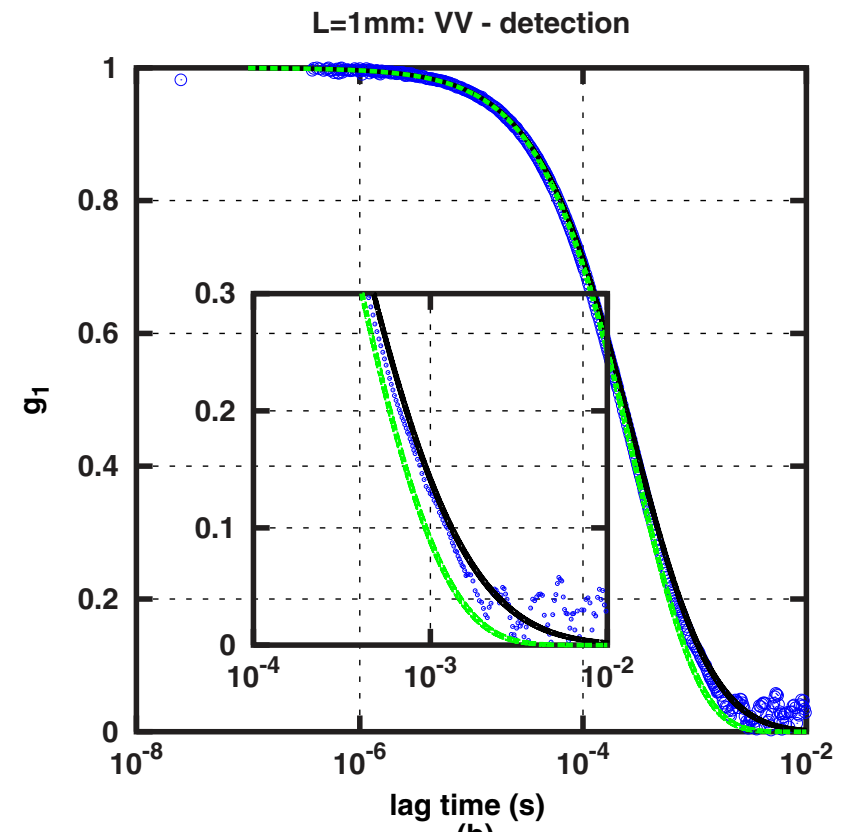

(b)

FIG. 2. (Color online) Measured and calculated autocorrelation functions $g_{1}(\tau)$ of light transmitted through 1-mm-thick PS454 sample when (a) cross-polarized and (b) copolarized light is detected. Markers represent measured $g_{1}(\tau)$, while dashed and solid lines are calculated with Eqs. (1) and (4), respectively. (a) Inset: Comparison of measured $g_{1}(\tau)$ obtained in cross-polarization (circles) and copolarization (asterisks) geometry. (b) Inset: The results at large lag times when copolarized light is considered.

suggests that for $L \geqslant 2 \mathrm{~mm}$ (i.e., $L / l^{*} \geqslant 6.7$ ), the polarization information is lost.

The simulated $g_{1}(\tau)$ based on $P_{F}(s)$ [i.e., using Eq. (1)] agree well with the measurements in the case of thick cuvettes ( $L=5$ and $10 \mathrm{~mm}$ ), suggesting that the continuum limit is a valid approximation for the experimental conditions (i.e., all detected light is diffuse). However, in the case of $L=1 \mathrm{~mm}$,

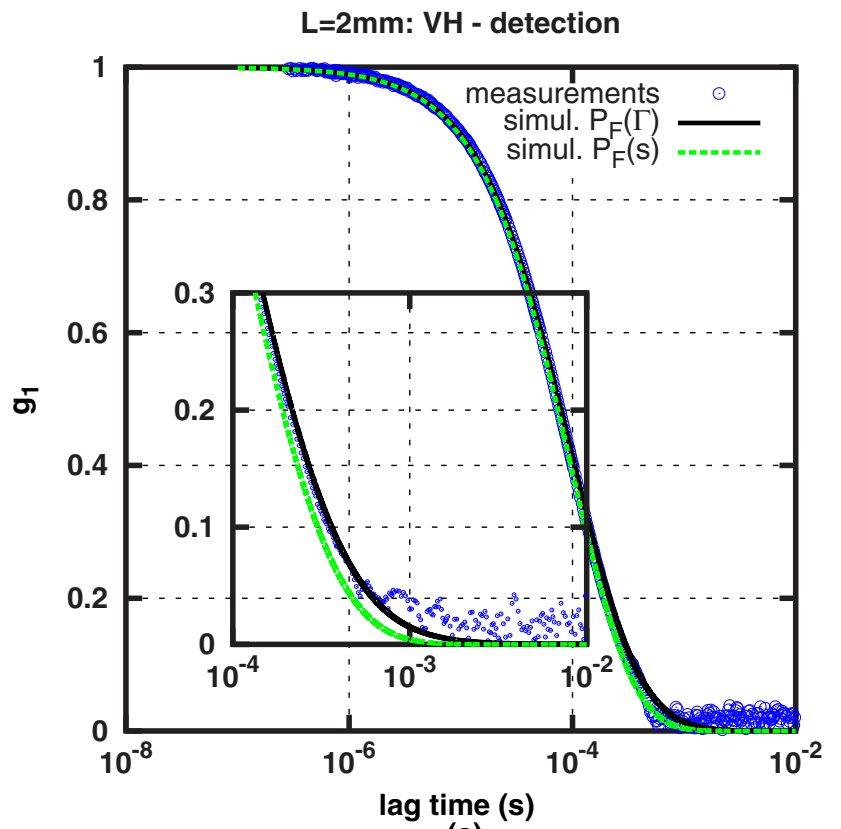

(a) the simulations based on $P_{F}(s)$ deviate from the measurements for both polarization directions. The same behavior is apparent for a $L=2 \mathrm{~mm}$ cuvette, but the disagreement starts at larger lag times $\tau$ that correspond to shorter light paths $s$ compared to the setup with a $L=1 \mathrm{~mm}$ cuvette. However, the results based on $P_{F}(\Gamma)$ agree well with the experimental data for all cuvette thicknesses $L$ and polarization channels. This suggests

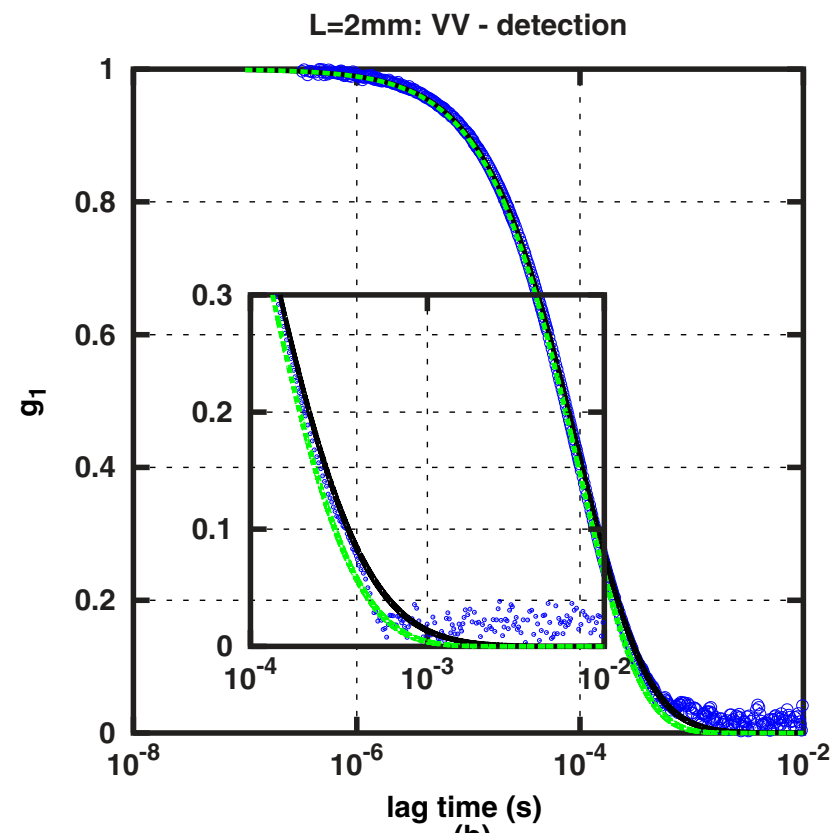

(b)

FIG. 3. (Color online) Measured and calculated autocorrelation functions $g_{1}(\tau)$ of light transmitted through 2-mm-thick PS454 sample when (a) cross-polarized and (b) copolarized light is detected. Markers represent measured $g_{1}(\tau)$, while dashed and solid lines are calculated with Eqs. (1) and (4), respectively. Insets: The results at large lag times when (a) cross-polarized and (b) copolarized light are considered. 


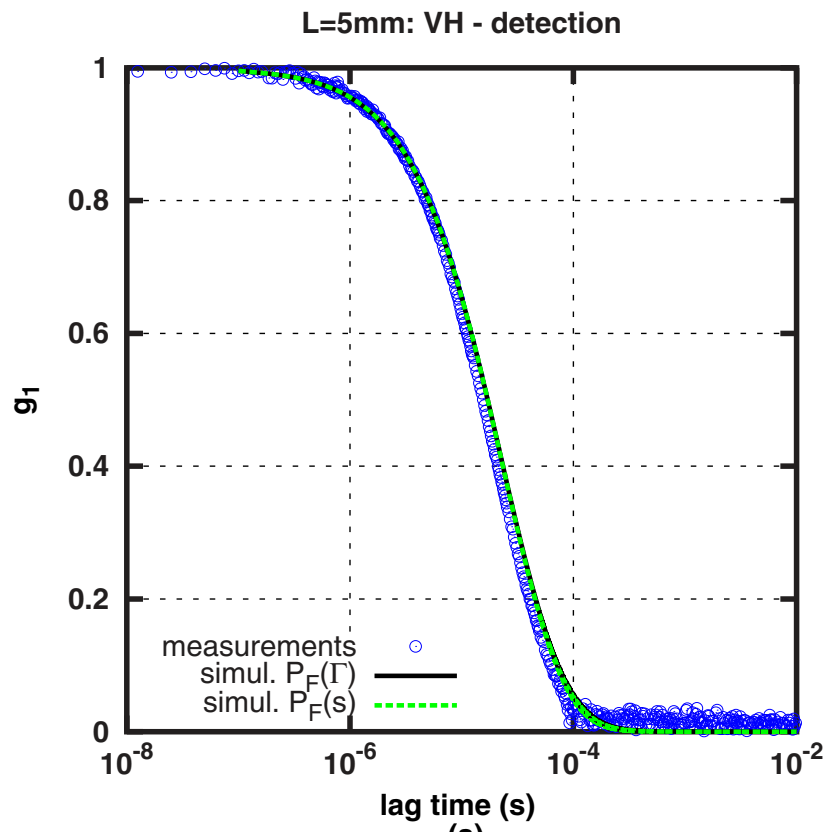

(a)

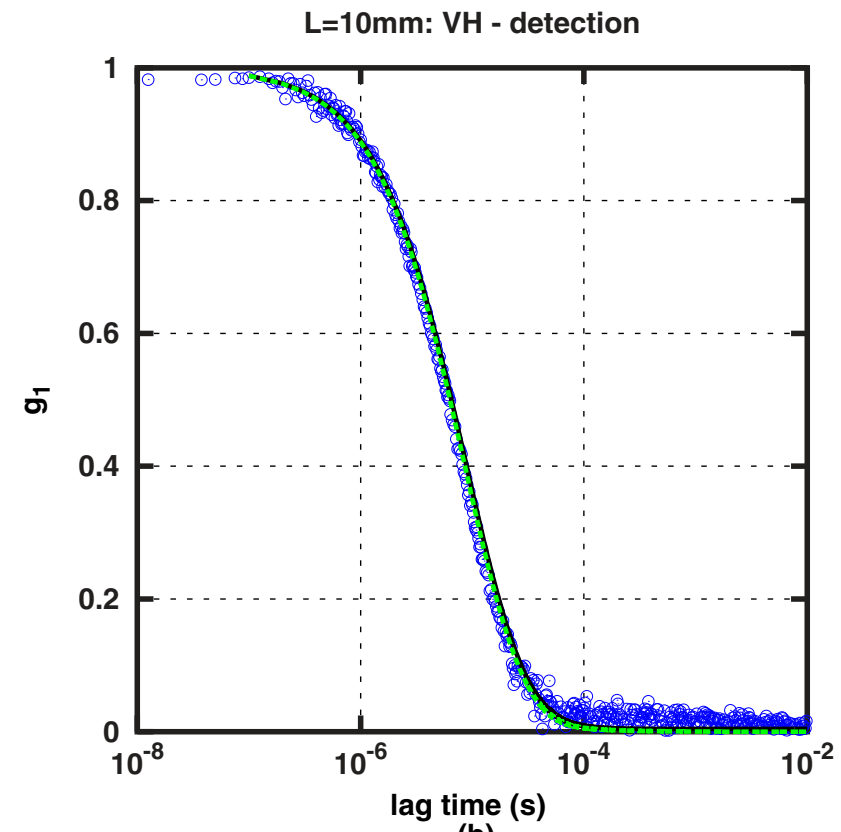

(b)

FIG. 4. (Color online) Measured and calculated autocorrelation functions $g_{1}(\tau)$ when cross-polarized light transmitted through (a) 5-mmthick and (b) 10-mm-thick PS454 samples is considered. Markers represent measured $g_{1}(\tau)$, while dashed and solid lines are calculated with Eqs. (1) and (4), respectively.

that for thin cuvettes, the continuum limit approximation is not valid. To explore this limit, we investigate the relation between $s$ and $\Gamma$ using a SCATTER3D setup where the cuvette is not modeled (i.e., matched refractive indices are employed) in order to avoid light reflections. The sample geometry is the same as the cuvette interior. This allows us to study the transition from single scattered to diffuse light transport caused exclusively by the scattering process in samples with different optical properties. Although the short trajectories of transmitted light have small $s, \Gamma$, and $y$, the linear relation $y_{\text {th }}=2 k^{2} s / l^{*}$ valid for diffuse light [21] is not expected. First, the relation between $s$ and $\Gamma$ is studied in order to find

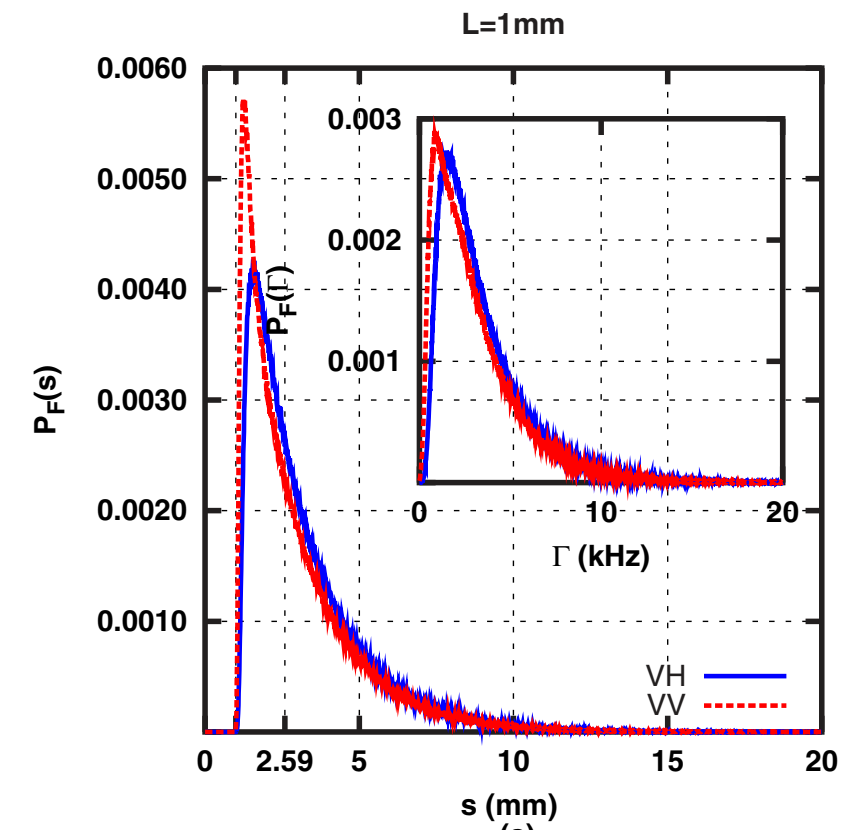

(a)

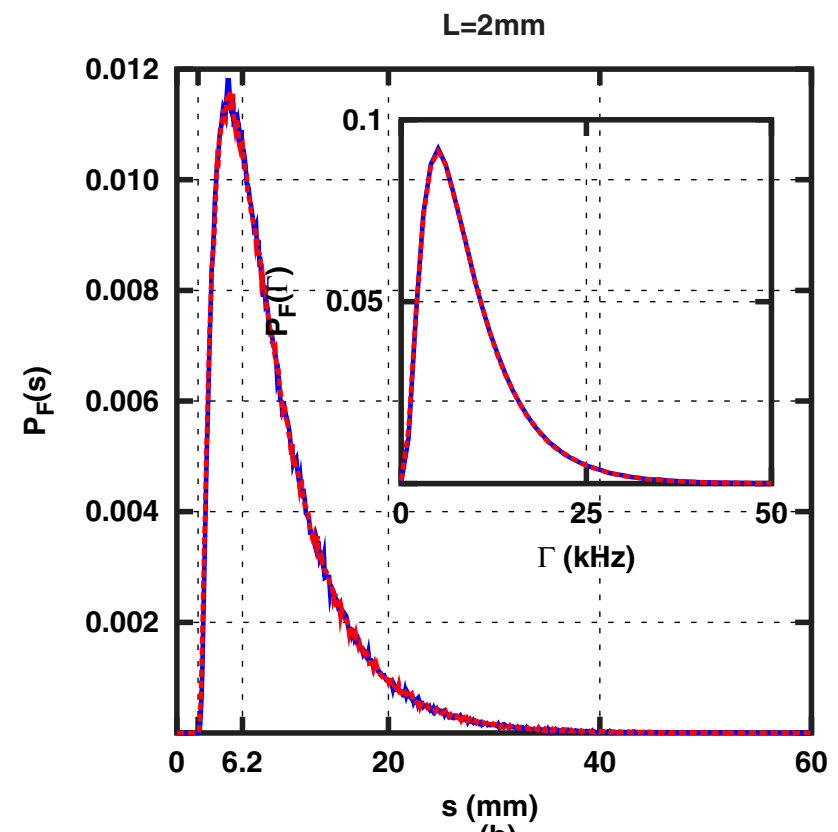

(b)

FIG. 5. (Color online) Distributions $P_{F}(s)$ for (a) $(L=1)$-mm-thick and (b) $(L=2)$-mm-thick PS454 samples when cross-polarized (solid) and copolarized (dashed) light are considered. The bin size is $0.01 \mathrm{~mm}(0.1 \mathrm{~mm})$ for $L=1 \mathrm{~mm}(L=2 \mathrm{~mm})$. Insets: Distributions $P_{F}(\Gamma)$ obtained from the same simulations as the $P_{F}(s)$ in the main figures. The bin size is $10 \mathrm{~Hz}(1000 \mathrm{~Hz})$ for $L=1 \mathrm{~mm}(L=2 \mathrm{~mm})$. Distributions with the same bin sizes are used to calculate autocorrelation functions in Figs. 2 and 3. 


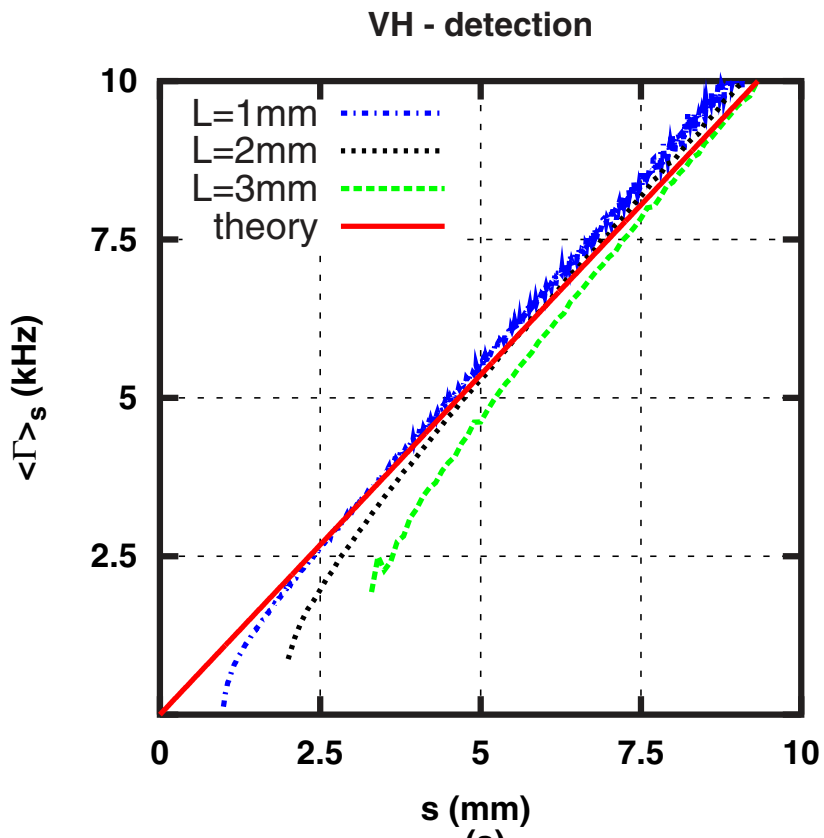

(a)

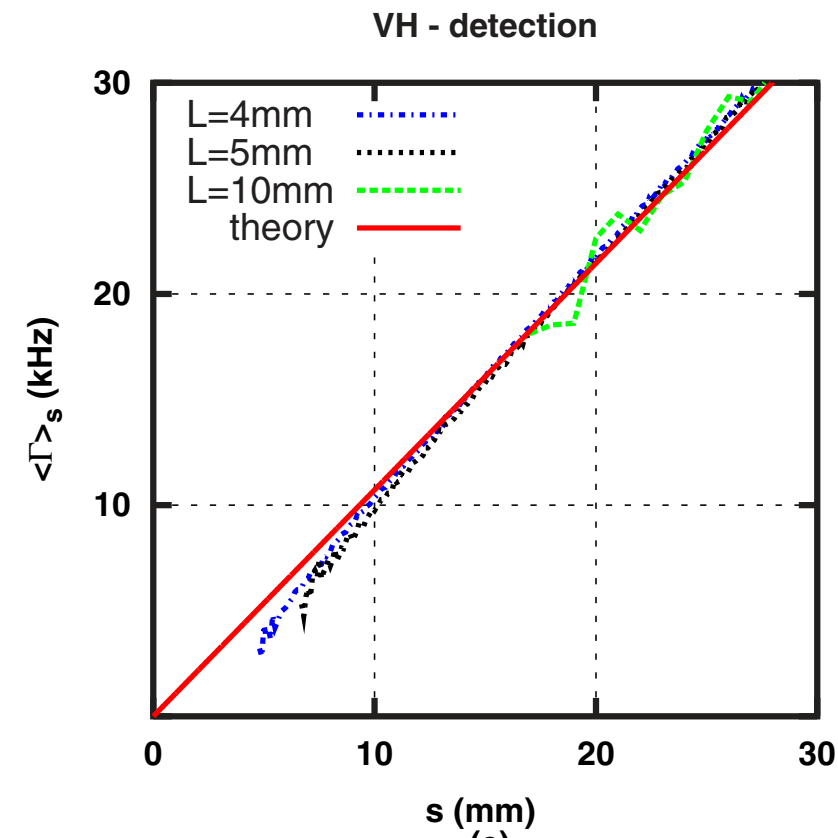

(c)

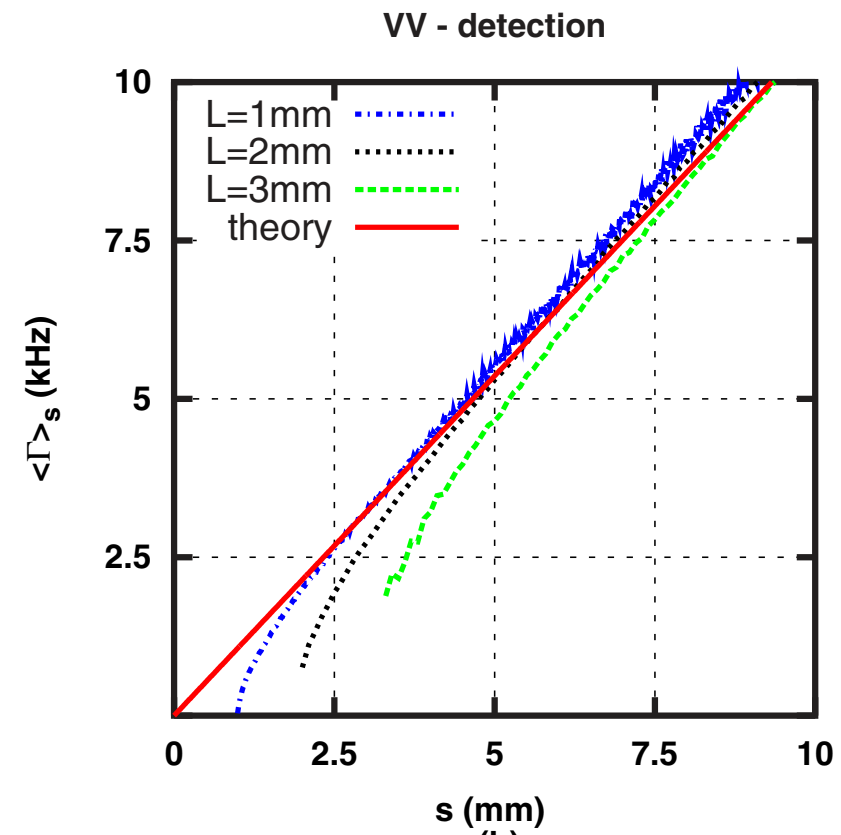

(b)

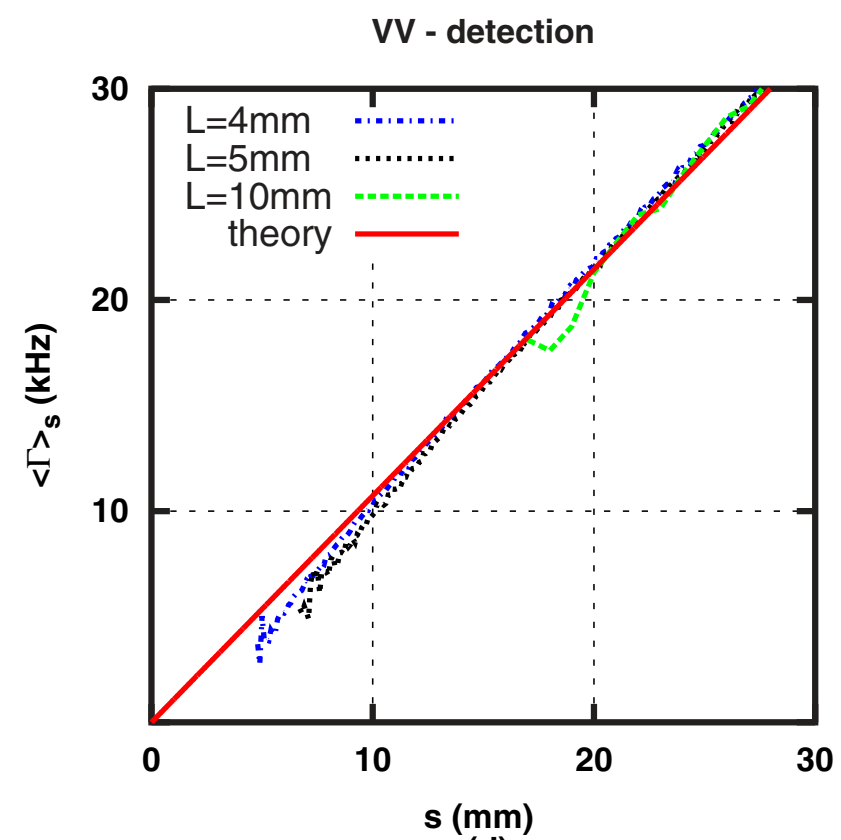

(d)

FIG. 6. (Color online) $\langle\Gamma\rangle_{s}$ calculated for PS454 as described in the text. The $P_{F}(s)$ bins are of the same sizes as those used to calculate $g_{1}(\tau)$ in Figs. 2-4. (a),(c) Results when cross-polarized light is detected, and (b),(d) results when copolarized light is detected. Solid red lines represent theoretically calculated $\Gamma_{\text {th }}=y_{\text {th }} D_{\text {eff }}=2 k^{2} s / l^{*} D_{\text {eff }}$.

the shortest path lengths of diffuse light trajectories $s_{\min }$. The simulated results for sample thicknesses $L=1$ to $10 \mathrm{~mm}$ are shown in Fig. 6. The solid lines are relations for diffuse light calculated as $\Gamma_{\text {th }}=y_{\text {th }} D_{\text {eff }}$. From all subplots in Fig. 6 , it is obvious that the relation between $\langle\Gamma\rangle_{s}$ and $s$ is similar for copolarized and cross-polarized light. Moreover, if we define $s_{\min }$ as the smallest $s$ value for which $\left(\Gamma_{\mathrm{th}}-\langle\Gamma\rangle_{s}\right) / \Gamma_{\mathrm{th}}<1 \%$, we find that $s_{\min }$ depends on $L / l^{*}$. This demonstrates that the ratio of weakly scattered to diffuse light depends on $L / l^{*}$. The relative contribution of diffuse light to the signal grows with $s$. The weakly scattered light dominates the signal composed of trajectories with path lengths $<s_{\min }=2.59 \mathrm{~mm}$ (1-mm-thick sample) and $<s_{\min }=6.2 \mathrm{~mm}$ (2-mm-thick sample). This is the most pronounced for a 1-mm-thick sample when copolarized light is detected [see Fig. 5(a)], thus resulting in the largest difference between $g_{1}(\tau)$ calculated using Eqs. (1) and (4). The comparison between Figs. 5(a) and 5(b) shows that the relative contribution of nondiffuse light trajectories is smaller for a 2-mm-thick sample. Therefore, $g_{1}(\tau)$ calculated using Eq. (1) for a 1-mm-thick sample start deviating from the measurements at larger values $\left(g_{1} \approx 0.5\right)$ [see Fig. 2(b)] than the corresponding $g_{1}(\tau)$ calculated for a 2-mm-thick sample 


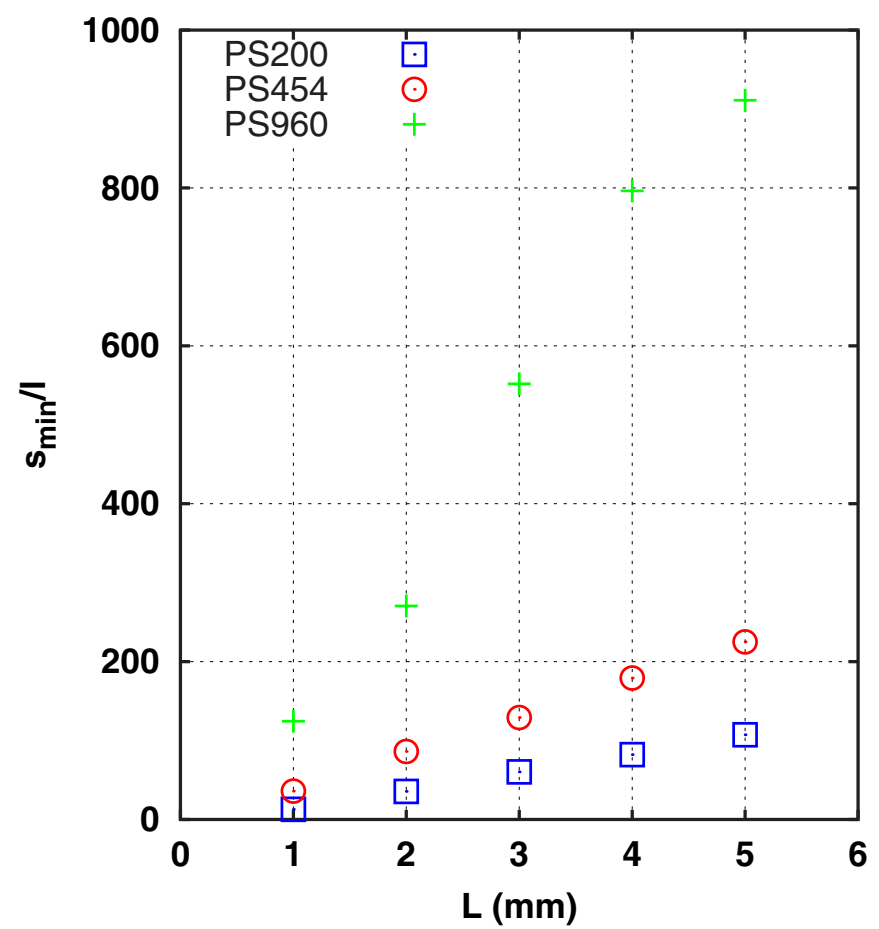

FIG. 7. (Color online) Lengths of the shortest light trajectories that can be considered diffuse. These data were determined as the simulated value of $s$ in Fig. 6 for which $\langle\Gamma\rangle_{s}$ starts to deviate from the theoretical value, i.e., the smallest $s$ for which $\left(\Gamma_{\mathrm{th}}-\langle\Gamma\rangle_{s}\right) / \Gamma_{\mathrm{th}}<1 \%$.

$\left(g_{1} \approx 0.3\right)$ [see Fig. 3(b)]. The contribution of nondiffuse light in a detected signal is even smaller for a 5-mm-thick sample, while for a 10 -mm-thick sample, the signal is composed of diffuse light only. The distributions for 5- and 10-mm-thick samples are not presented due to limited number of pages.

The results for PS200 and PS960 serve as a further validation and are presented in the Appendix. Here, we only present the results of the shortest diffuse light trajectories that were obtained as described above. The results are summarized in Fig. 7. The length of the shortest diffuse light trajectories $s_{\text {min }}$ is normalized to the scattering mean free path of the corresponding sample. It can be seen that $s_{\min } / l$ grows with $L$ and particle size (i.e., $g_{\text {eff }}$ ). Due to limited number of pages, we cannot present all simulated $g_{1}(\tau)$ using Eqs. (1) and (4) for the different $L$, but we note here that the smallest $L$ to ensure diffuse transport of light [i.e., simulated $g_{1}(\tau)$ obtained using Eqs. (1) and (4) overlap over the whole range of lag time] is $4 \mathrm{~mm}$ (PS200), $3 \mathrm{~mm}$ (PS454), and $2 \mathrm{~mm}$ (PS960). Therefore, the smallest $L / l^{*}$ for which all transmitted light is diffuse is 14 (PS200), 10 (PS454), and 7 (PS960); i.e., $L / l=18$ (PS200), $L / l=42$ (PS454), and $L / l=74$ (PS960). Although the largest difference between $g_{1}(\tau)$ of copolarized and cross-polarized light is for PS200 [see, e.g., inset in Fig. 8(b)] among all considered samples, the linear polarization is best preserved when light is scattered on the largest particles (i.e., PS960). First, the comparison between $g_{1}(\tau)$ of copolarized and cross-polarized light calculated using Eq. (4) (or between the corresponding measurements) shows that the linearly polarized light depolarizes before it becomes diffuse; i.e., the smallest $L / l^{*}$ for which $g_{1}(\tau)$ of copolarized and cross-polarized transmitted light overlap over the whole range of lag time is 10 (PS200), 7 (PS454), and 7 (PS960). This corresponds to $L / l=14$ (PS200), $L / l=28$ (PS454), and $L / l=74$ (PS960). Therefore, the number of scattering events required for depolarization grows with the size of scattering
L=1mm: VH - detection

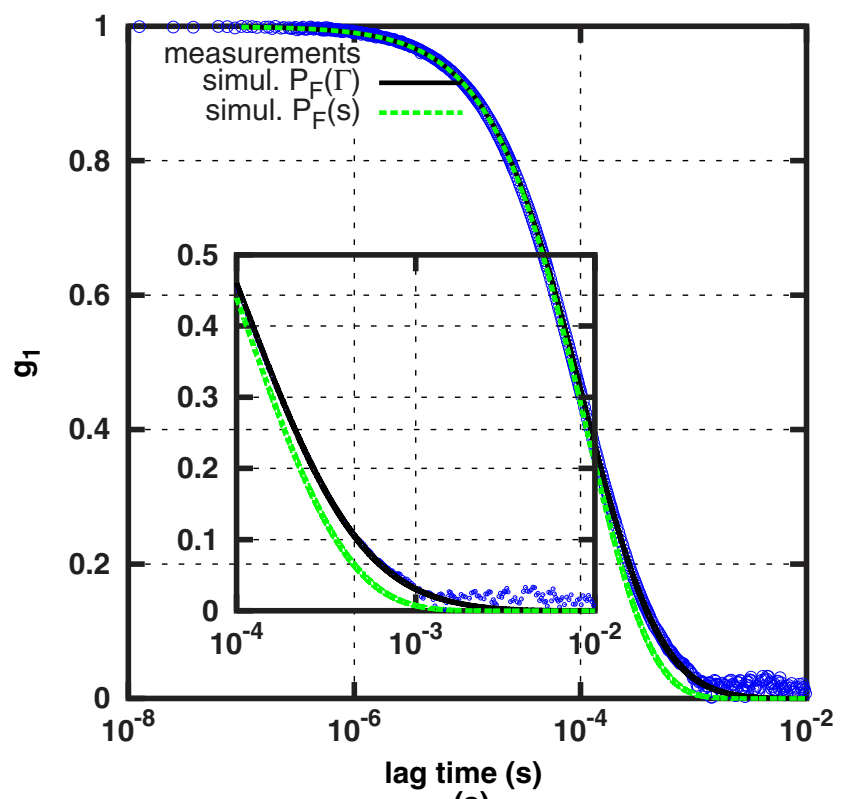

(a)

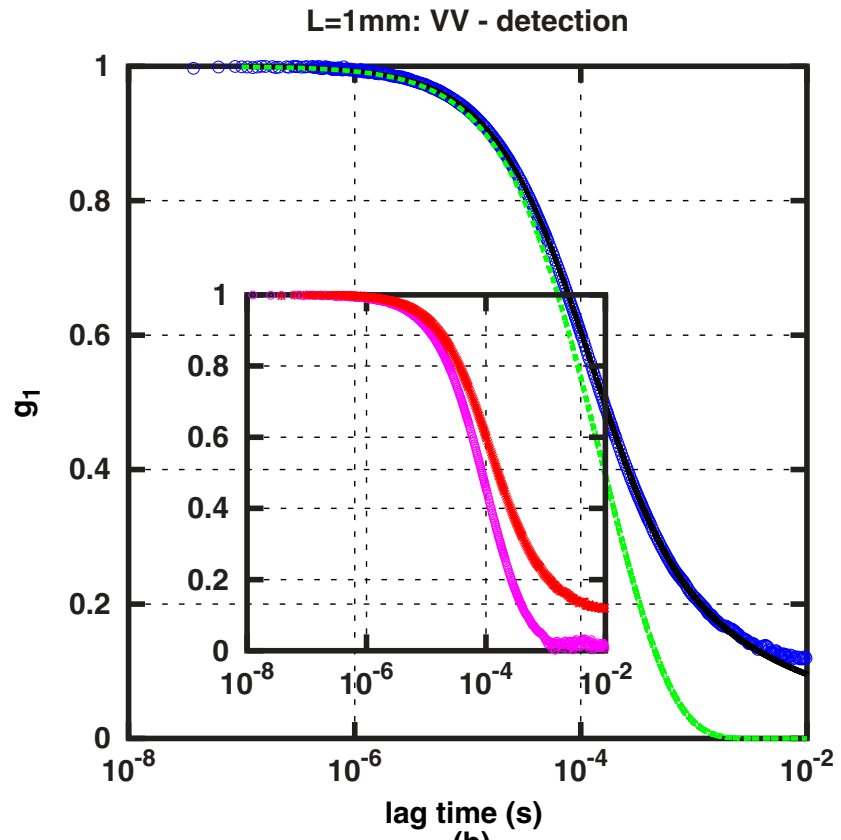

(b)

FIG. 8. (Color online) Measured and calculated autocorrelation functions $g_{1}(\tau)$ of light transmitted through 1-mm-thick PS200 sample when (a) cross-polarized and (b) copolarized light is detected. Markers represent measured $g_{1}(\tau)$, while dashed and solid lines are calculated with Eqs. (1) and (4), respectively. (a) Inset: The results at large lag times when cross-polarized light is considered. (b) Inset: $g_{1}(\tau)$ when cross-polarized (circles) and copolarized (asterisks) light are measured. 
particles. Note, however, that the sample thickness resolution of $1 \mathrm{~mm}$ is not sufficient, thus resulting in the same minimum $L / l^{*}$ necessary to depolarize and diffuse light transmitted through the PS960 sample. However, the same conclusion as for light transmission through PS200 and PS454 can be expected even when the PS960 sample is used, if the samples with intermediate thicknesses are considered.

\section{CONCLUSIONS}

The electric field autocorrelation functions $g_{1}(\tau)$ of light transmitted through three samples with different size of scattering particles and four cuvettes of different thicknesses are measured and obtained with Monte Carlo simulations. For the two thinnest cuvettes, the simulation results deviated from the measurements at large lag times if the path-length distributions $P_{F}(s)$ were used to calculate $g_{1}(\tau)$. The deviations were even more pronounced when copolarized instead of cross-polarized light was detected. The $g_{1}(\tau)$ calculated using distributions based on the sums of square momentum transfers $P_{F}(\Gamma)$ accurately predict measurements over the whole range of lag times for all samples, cuvettes, and polarization channels considered. Both distributions were obtained from the same Monte Carlo simulations. Therefore, it is concluded that the deviations are caused by employing the continuum limit approximation $(s=n l)$ when using $P_{F}(s)$ to calculate $g_{1}(\tau)$. The shape of $g_{1}(\tau)$ at large lag times is determined by the short light trajectories, while the continuum limit can be assumed for diffuse light. The path lengths and sums of the square momentum transfers are linearly dependent if the light propagation is diffuse. On the other hand, we showed that the path lengths of short light trajectories are nonlinearly related to the corresponding sums of square momentum transfers. From this relation, we estimated for which light paths the continuum limit can be assumed. In this manner, the shortest diffuse light trajectories $s_{\min }$ were determined. We furthermore showed that $s_{\text {min }}$ grows with the sample thickness and scattering anisotropy. We also showed that the minimum $L / l$ necessary to detect only diffuse light grows with the scattering anisotropy. Finally, it is shown that linearly polarized illumination is depolarized before it becomes diffuse, no matter the sample considered, and that polarization is preserved longer when light is scattered on larger particles. Note that whether the light trajectories are diffuse depends on their shape, which is captured by the sum of the squared momentum transfers that directly depends on the scattering angles contained in the light path. On the other hand, the distribution $P_{F}(s)$ does not distinguish between the photon trajectories of the same length but having very different shapes (different sums of the square momentum transfers), which are typical for weakly scattered light.

The question of whether the continuum limit can be passed on some of the nondiffuse photon trajectories that might be sufficiently long to contain large enough number of scattering events, so that $s=n l$ is satisfied, is a subject of future work.

We hope that the findings presented in this paper, in particular those regarding the length of the shortest diffuse light trajectories, will help researchers, specifically in the field of biomedical optics. It can also help sample preparation for diffusing wave spectroscopy, since either the optimal sample turbidity or cell thickness can now be determined. Finally, we would like to mention that while our experiments focused on diffusing wave spectroscopy, the results also apply to electromagnetic waves in general, as long as the radiative transfer theory is valid.

\section{ACKNOWLEDGMENTS}

Miloš Šormaz acknowledges financial support by The Commission for Technology and Innovation (CTI), Switzerland (Project No. 14313.1 PFNM-NM). Ursina Caluori from EMPA is acknowledged for the help in performing numerical simulations.

\section{APPENDIX}

Here, the electric field autocorrelation functions $g_{1}(\tau)$ for PS200 and PS960 are presented as a further validation of SCATTER3D. In particular, it is shown that the drawn conclusions are valid, independent of the particle size. Distributions and figures showing $\langle\Gamma\rangle_{s}$ vs $s$ are not shown due to limited number of pages. However, the results for the lengths of the shortest diffuse light trajectories $s_{\min }$ are shown in Fig. 7. The results for PS200 are presented in Fig. $8(L=1 \mathrm{~mm})$, Fig. $9(L=2 \mathrm{~mm})$, and Fig. $10(L=5$ and $L=10 \mathrm{~mm})$. Although all three samples have the same $l^{*}$, the largest difference between $g_{1}(\tau)$ for copolarized light calculated using Eqs. (1) and (4) is due to a large relative contribution of light with weakly corrugated trajectories (the light with small $\Gamma)$. Note that PS200 has the smallest scattering coefficient. The results for PS960 are presented in Fig. $11(L=1 \mathrm{~mm})$, Fig. $12(L=2 \mathrm{~mm})$, and Fig. $13(L=5$ and $L=10 \mathrm{~mm})$. The inertia of scattering particles and fluid [26] are not captured in Monte Carlo simulations. The effect is more pronounced for larger scattering particles and larger $L / l^{*}$. Therefore, the measured $g_{1}(\tau)$ for PS960 are "corrected" for the inertia effects of scattering particles and fluid in order to compare them to simulations. As expected, the largest discrepancy between simulated and measured $g_{1}(\tau)$ is for a $L=10 \mathrm{~mm}$ cuvette at short lag times defined by long light trajectories. The difference between measurements and corrected $g_{1}(\tau)$ is negligible for $L=1$ and $L=2 \mathrm{~mm}$ cuvettes. Next, the procedure for correcting measurements is described. First, the mean-square displacement of scattering particles $\left\langle\Delta x^{2}(\tau)\right\rangle$ is extracted from the measured $g_{1}(\tau)$ using the expression

$$
g_{1}(\tau)=\frac{\left(\frac{L}{l^{*}}+\frac{4}{3}\right) k \sqrt{\left\langle\Delta x^{2}(\tau)\right\rangle}}{\left[1+\frac{4}{9} k^{2}\left\langle\Delta x^{2}(\tau)\right\rangle\right] \sinh \left[\frac{L}{l^{*}} k \sqrt{\left\langle\Delta x^{2}(\tau)\right\rangle}\right]+\frac{4}{3} k \sqrt{\left\langle\Delta x^{2}(\tau)\right\rangle} \cosh \left[\frac{L}{l^{*}} k \sqrt{\left\langle\Delta x^{2}\right\rangle}\right]} .
$$

Note that Eq. (A1) assumes laterally infinite cuvette and illumination, which is not the case for the experimental conditions considered here. However, the cuvettes $L=1$ and $L=2 \mathrm{~mm}$ mimic the aforementioned conditions well. On 


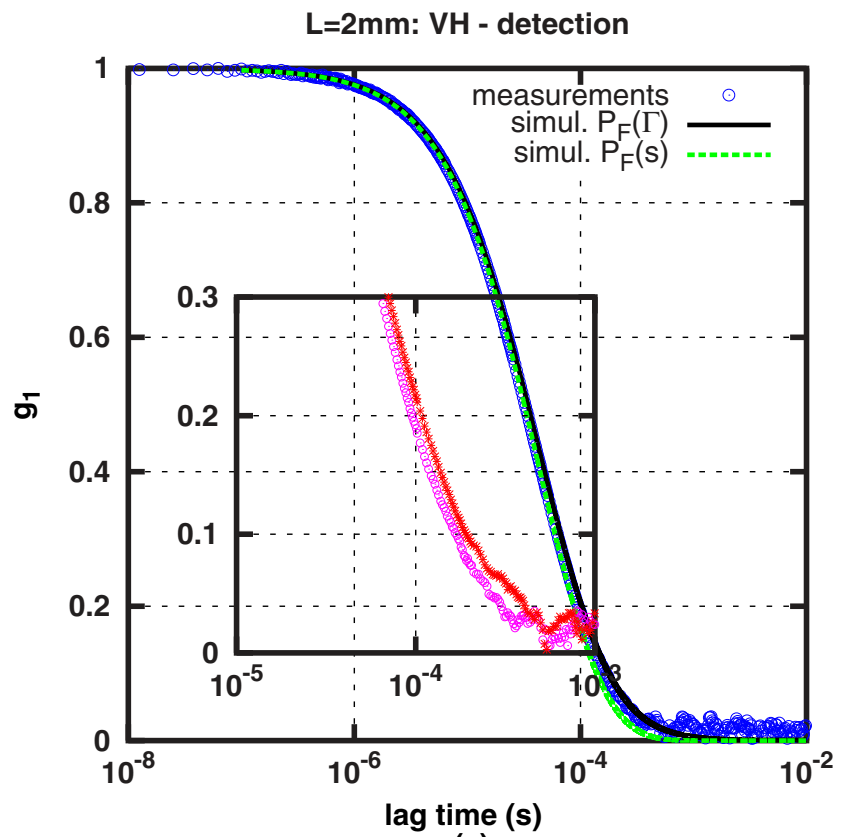

(a)

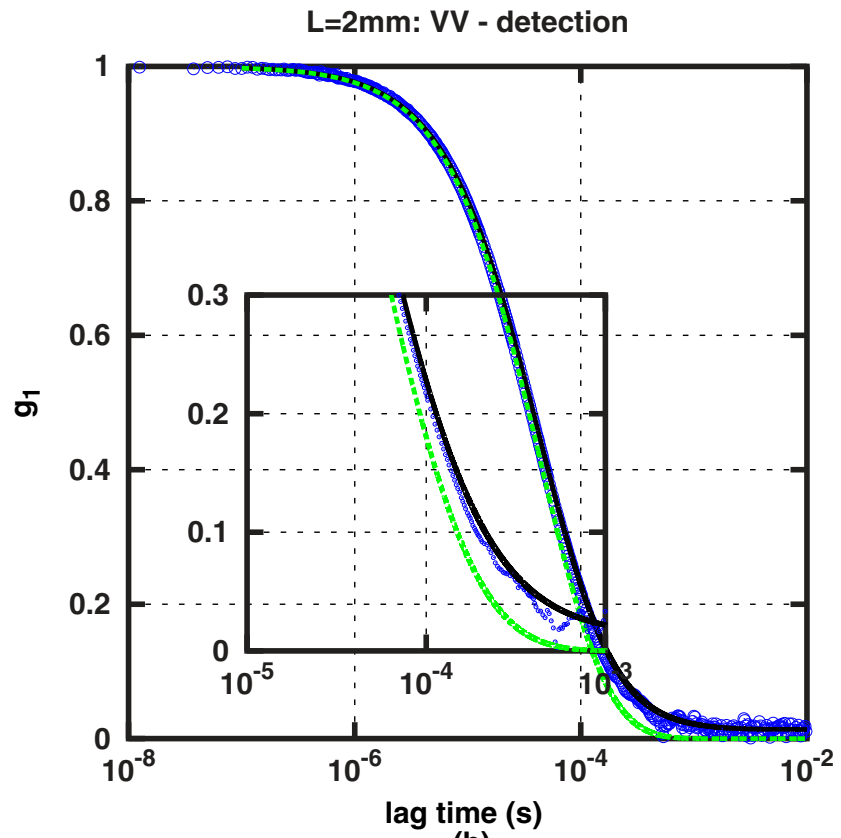

(b)

FIG. 9. (Color online) Measured and calculated autocorrelation functions $g_{1}(\tau)$ of light transmitted through 2-mm-thick PS200 sample when (a) cross-polarized and (b) copolarized light is detected. Markers represent measured $g_{1}(\tau)$, while dashed and solid lines are calculated with Eqs. (1) and (4), respectively. (a) Inset: $g_{1}(\tau)$ when cross-polarized (circles) and copolarized (asterisks) light are measured. (b) Inset: The results at large lag times when copolarized light is considered.

the other hand, Eq. (A1) also assumes diffuse light which is detected, regardless of the sample used, when light is transmitted through $L=5$ and $L=10 \mathrm{~mm}$ cuvettes. The extracted $\left\langle\Delta x^{2}(\tau)\right\rangle$ represents the mean-square displacement of scattering particles including the inertia effects. Next, the mean-square displacement of scattering particles not affected by inertia effects $\left\langle\Delta x^{2}(\tau)\right\rangle^{\prime}$ is calculated from [26]

$$
\frac{\left\langle\Delta x^{2}(\tau)\right\rangle}{\left\langle\Delta x^{2}(\tau)\right\rangle^{\prime}}=1-2 \sqrt{\frac{1}{\pi} \frac{\tau_{f}}{\tau}}+\frac{8}{9} \frac{\tau_{f}}{\tau}-\frac{\tau_{p}}{\tau}+\Xi\left(\frac{\tau_{p}}{\tau_{f}}, \frac{\tau}{\tau_{f}}\right) .
$$

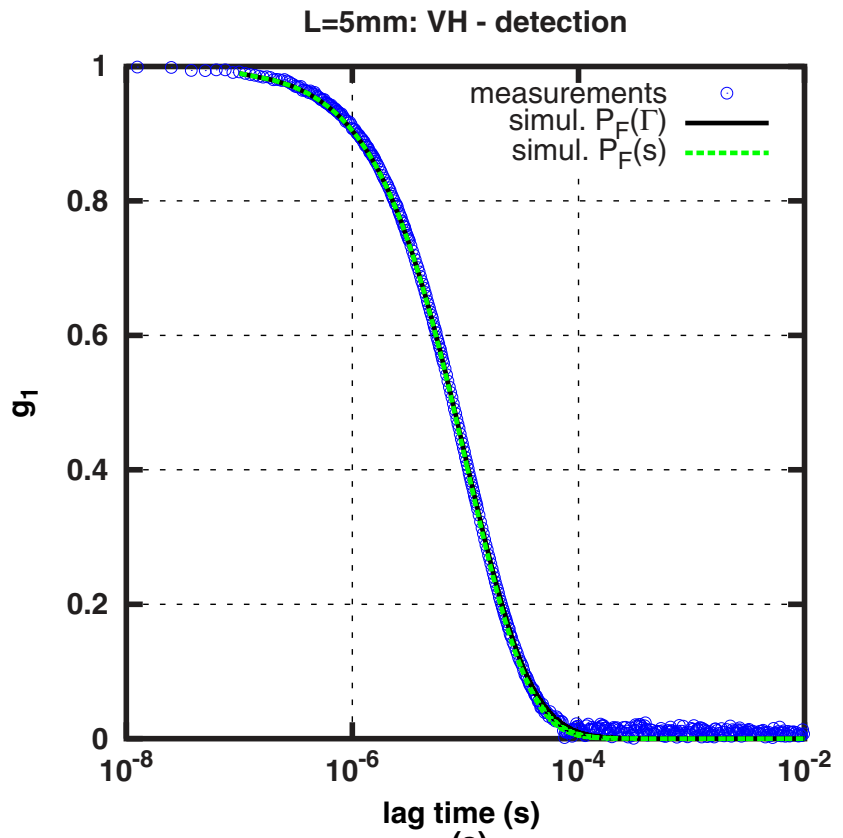

(a)

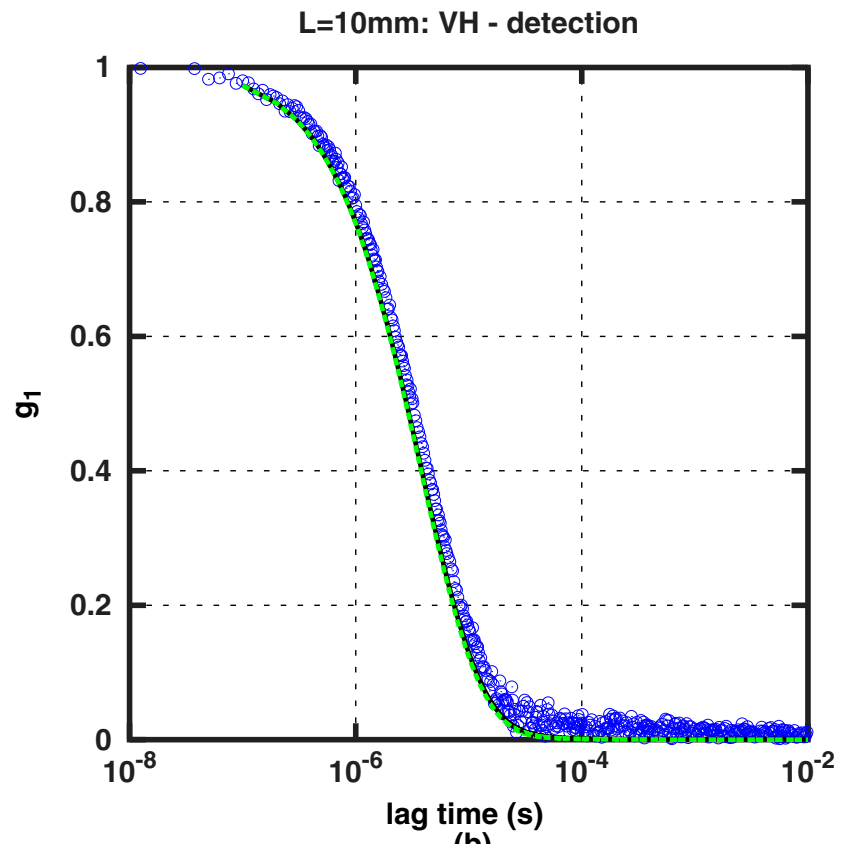

(b)

FIG. 10. (Color online) Measured and calculated autocorrelation functions $g_{1}(\tau)$ when cross-polarized light transmitted through (a) 5-mmthick and (b) 10-mm-thick PS200 samples is considered. Markers represent measured $g_{1}(\tau)$, while dashed and solid lines are calculated with Eqs. (1) and (4), respectively. 


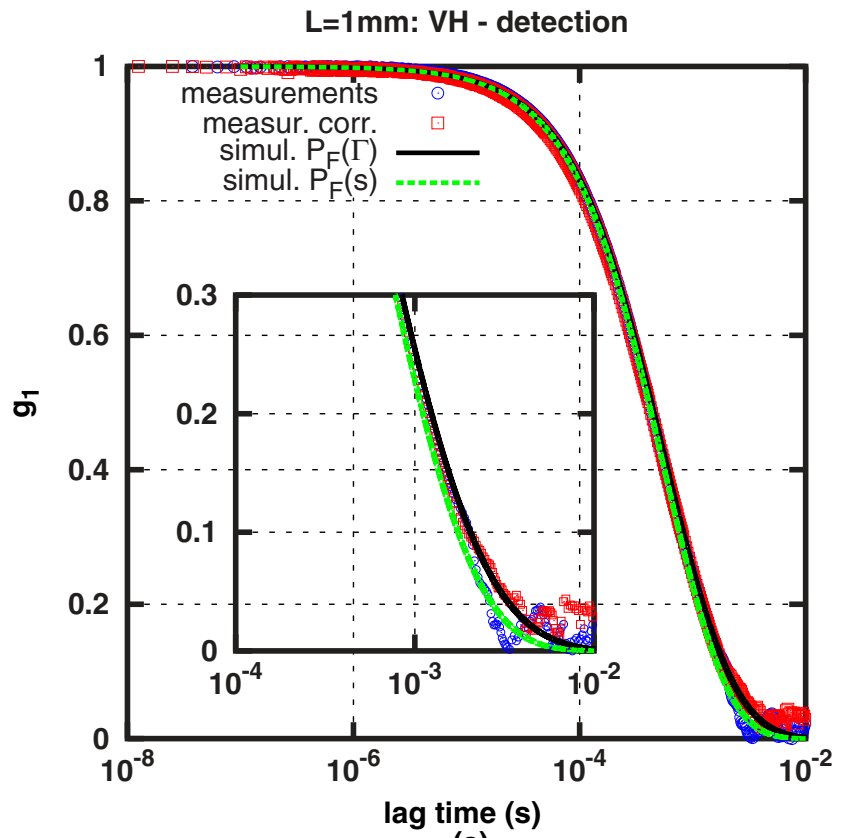

(a)

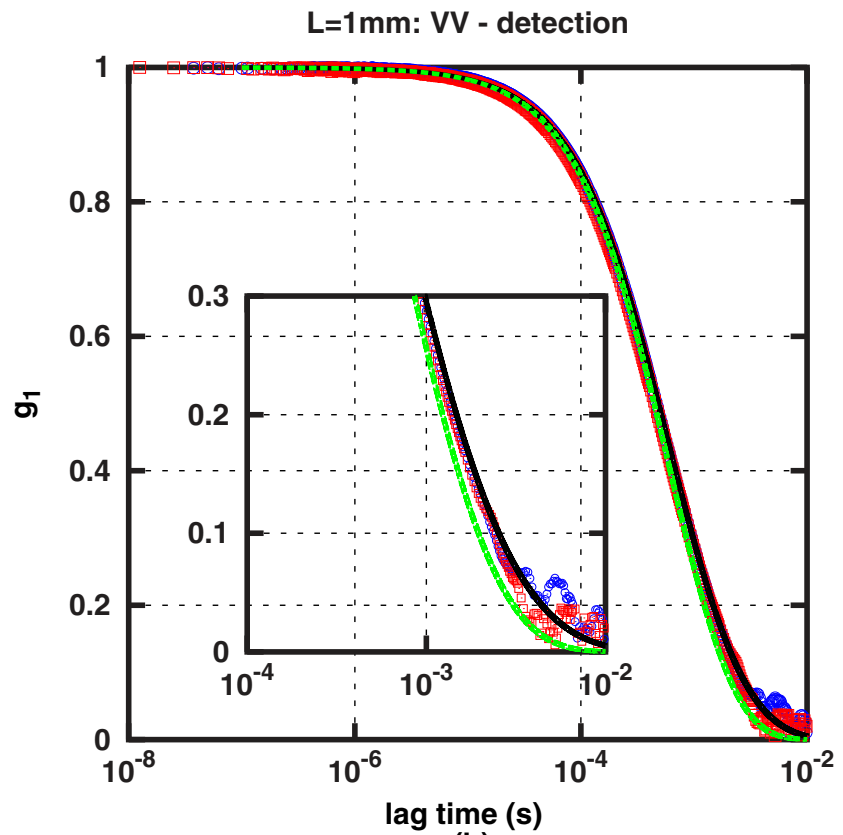

(b)

FIG. 11. (Color online) Measured and calculated autocorrelation functions $g_{1}(\tau)$ of light transmitted through 1-mm-thick PS960 sample when (a) cross-polarized and (b) copolarized light is detected. Markers represent measured (circles) and corrected measured (squares) $g_{1}(\tau)$, while dashed and solid lines are calculated with Eqs. (1) and (4), respectively. Insets: The results at large lag times.

Here, inertia effects of scattering particles and fluid are represented by the characteristic times $\tau_{p}=\frac{2}{9} \bar{r}^{2} \rho_{p} / \eta$ and $\tau_{f}=\bar{r}^{2} \rho_{f} / \eta$, respectively. The characteristic time $\tau_{f}$ is the time necessary for a perturbed flow field to diffuse a distance of one particle's radius. Note that the fluid perturbation due to the motion of a scattering particle is not captured by the "standard"
Langevin equation. The small correction term $\Xi\left(\frac{\tau_{p}}{\tau_{\tau}}, \frac{\tau}{\tau_{f}}\right)$ [26] is neglected in this work. The densities of scattering particles $\rho_{p}=1060 \mathrm{~kg} / \mathrm{m}^{3}$ and water $\rho_{f}=1000 \mathrm{~kg} / \mathrm{m}^{3}$ are used. Finally, the corrected $g_{1}(\tau)$ is calculated using Eq. (A1) with $\left\langle\Delta x^{2}\right\rangle^{\prime}$ as an input. The largest difference between $g_{1}(\tau)$ calculated using Eqs. (1) and (4) is for a $L=1 \mathrm{~mm}$ cuvette

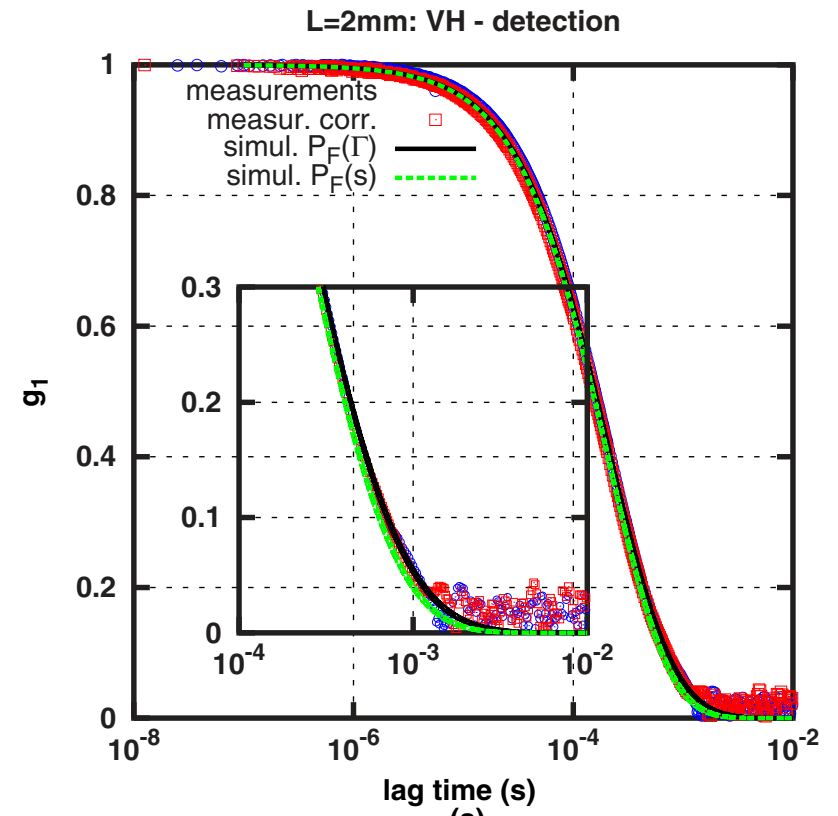

(a)

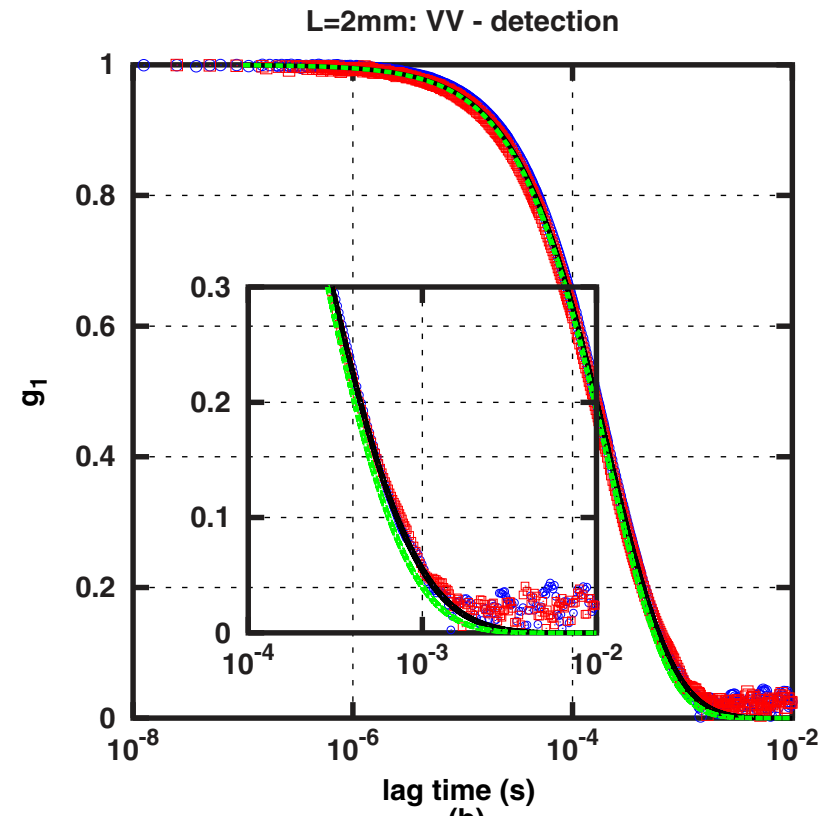

(b)

FIG. 12. (Color online) Measured and calculated autocorrelation functions $g_{1}(\tau)$ of light transmitted through 2-mm-thick PS960 sample when (a) cross-polarized and (b) copolarized light is detected. Markers represent measured (circles) and corrected measured (squares) $g_{1}(\tau)$, while dashed and solid lines are calculated with Eqs. (1) and (4), respectively. Insets: The results at large lag times. 


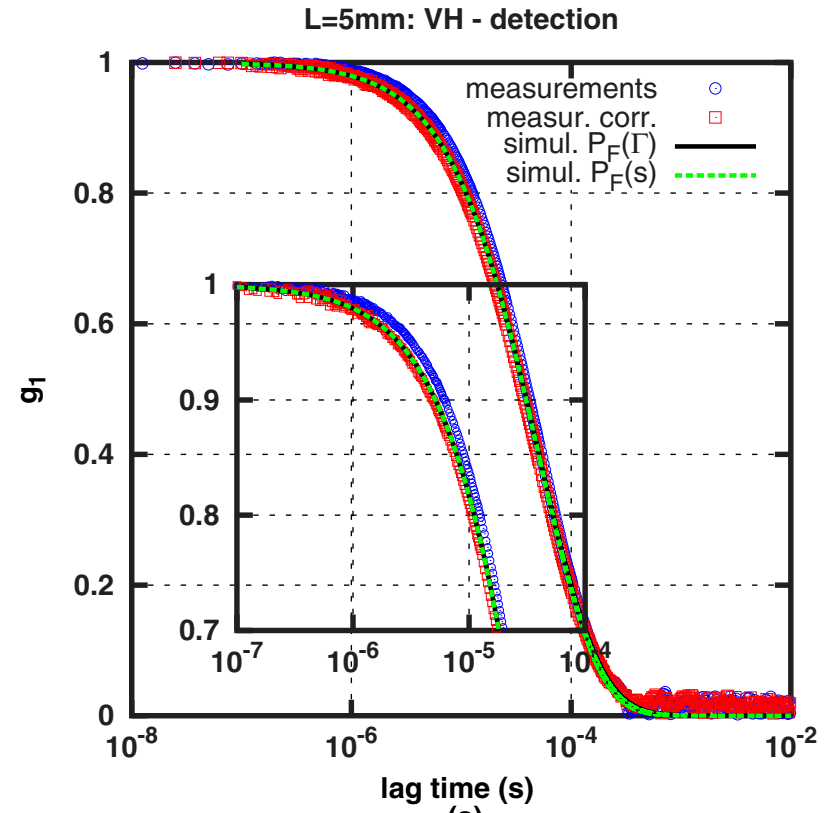

(a)

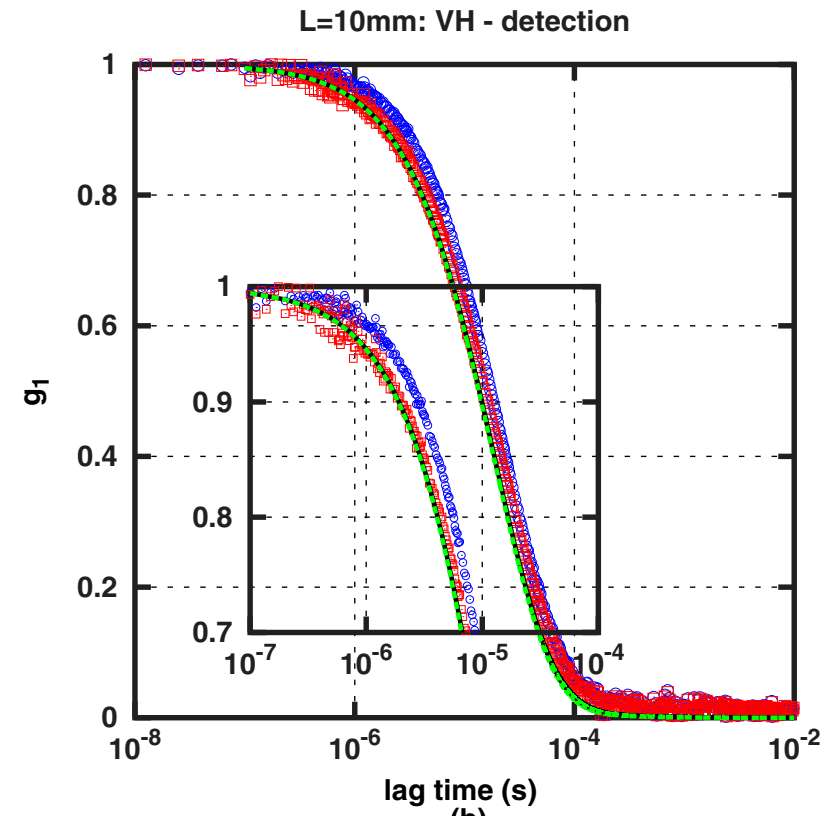

(b)

FIG. 13. (Color online) Measured and calculated autocorrelation functions $g_{1}(\tau)$ when cross-polarized light transmitted through (a) 5-mmthick and (b) 10-mm-thick PS960 samples is considered. Markers represent measured (circles) and corrected measured (squares) $g_{1}(\tau)$, while dashed and solid lines are calculated with Eqs. (1) and (4), respectively. Insets: The results at short lag times.

when copolarized light is detected. By comparing the results for, e.g., a $L=1 \mathrm{~mm}$ cuvette among all three samples, it can be concluded that $g_{1}(\tau)$ are decaying at the shortest lag times for PS200 due to the largest $D_{\text {eff }}$.
[1] A. Ishimaru, App. Opt. 28, 2210 (1989).

[2] A. Knuttel, J. M. Schmitt, and J. R. Knutson, App. Opt. 32, 381 (1993).

[3] D. A. Boas, M. A. O'Leary, B. Chance, and A. G. Yodh, Proc. Natl. Acad. Sci. (USA) 91, 4887 (1994).

[4] F. C. MacKintosh, J. X. Zhu, D. J. Pine, and D. A. Weitz, Phys. Rev. B 45, 8165 (1992).

[5] D. J. Pine, D. A. Weitz, P. M. Chaikin, and E. Herbolzheimer, Phys. Rev. Lett. 60, 1134 (1988).

[6] D. J. Pine, D. A. Weitz, J. X. Zhu, and E. Herbolzheimer, J. Phys. France 51, 2101 (1990).

[7] G. Maret and P. E. Wolf, Z. Phys. B 65, 409 (1987).

[8] G. Maret, Current Opinion Coll. Interf. Sci. 2, 251 (1997).

[9] D. A. Weitz, D. J. Pine, P. N. Pusey, and R. J. A. Tough, Phys. Rev. Lett. 63, 1747 (1989).

[10] F. C. MacKintosh and S. John, Phys. Rev. B 40, 2383 (1989).

[11] D. J. Durian, D. A. Weitz, and D. J. Pine, Science 252, 686 (1991).

[12] F. Jaillon, S. E. Skipetrov, J. Li, G. Dietsche, G. Maret, and T. Gisler, Opt. Exp. 14, 10181 (2006).

[13] S. Ulyanov, Phys. Rev. E 72, 052902 (2005).

[14] T. G. Mason, H. Gang, and D. A. Weitz, J. Opt. Soc. Am. A. 14, 139 (1997)
[15] F. Scheffold, P. Diaz-Leyva, M. Reufer, N. Ben Braham, I. Lynch and J. L. Harden, Phys. Rev. Lett. 104, 128304 (2010).

[16] J. Galvan-Miyoshi, J. Delgado, and R. Castillo, Eur. Phys. J. E 26, 369 (2008).

[17] M. Ninck, M. Untenberger, and T. Gisler, Biomed. Opt. Exp. 1, 1502 (2010).

[18] P.-A. Lemieux, M. U. Vera, and D. J. Durian, Phys. Rev. E 57, 4498 (1998).

[19] R. Carminati, R. Elaloufi, and J.-J. Greffet, Phys. Rev. Lett. 92, 213903 (2004).

[20] P. D. Kaplan, M. H. Kao, A. G. Yodh, and D. J. Pine, App. Opt. 32, 3828 (1993).

[21] A. A. Middleton and D. S. Fisher, Phys. Rev. B 43, 5934 (1991).

[22] M. Šormaz and P. Jenny, J. Opt. Soc. Am. A 29, 2174 (2012).

[23] M. Šormaz, T. Stamm and P. Jenny, J. Opt. Soc. Am. A 27, 1100 (2010).

[24] D. A. Weitz, J. X. Zhu, D. J. Durian, H. Gang, and D. J. Pine, Phys. Scr. T49, 610 (1993).

[25] J. C. Ramella-Roman, S. A. Prahl, and S. L. Jacques, Opt. Exp. 13, 10392 (2005).

[26] B. Lukić, S. Jeney, C. Tischer, A. J. Kulik, L. Forro, and E.-L. Florin, Phys. Rev. Lett. 95, 160601 (2005). 